association was found between birth weight SDS and the serum levels of IL6 $(p=47)$ or TNF $\alpha(p=30)$ in the first trimester of the pregnancy. However, good correlations were found between IL6 serum levels and TNF $\alpha($ Spearman rho $=0.24, p<0.005)$ and between IL6 levels and DAS28 (Spearman rho $=0.42, \mathrm{p}<0.001$ ) and CRP (Spearman rho=0.42, p<0.001).

Conclusion Elevated cytokine levels in the first trimester of women with RA are not associated with low birth weight. Therefore, this study does not provide a rationale for interfering with circulating cytokine levels solely to improve birth weight. Our conclusion contradicts previous literature which describes a negative association of cytokine levels in the third trimester with birth weight. For that reason, cytokine levels will also be determined in our cohort in the last trimester.

These data will also be presented at the 30th EWRR.

\title{
A159 ARE CIRCULATING PROINFLAMMATORY CYTOKINES ASSOCIATED WITH LOWER BIRTH WEIGHT OF NEWBORNS FROM WOMEN WITH RHEUMATOID ARTHRITIS?
}

F D 0 de Steenwinkel, J M W Hazes, Y A de Man, A C S Hokken, Y B de Rijke, R J E M Dolhain University Medical Center Rotterdam, Rotterdam, The Netherlands

10.1136/ard.2010.129650j

Background Recent studies have shown that high disease activity during pregnancy in women with rheumatoid arthritis (RA) is associated with lower birth weight of the child, even after adjustment for gestational age and medication. Lower birth weight, even within the normal range, has been linked with cardiovascular diseases and metabolic syndromes in adulthood. In studies on a limited number of healthy pregnant women, a role for interleukin 6 (IL6) and tumour necrosis factor $\alpha$ (TNF $\alpha$ ) in intrauterine growth restriction has been suggested. It is important to know whether the same holds true for RA during pregnancy, especially since the use of TNF-blockers seems to be safe during pregnancy.

Objectives To asses the levels of IL6 and TNF $\alpha$ during pregnancy in RA patients and to evaluate the correlations between these levels and the birth weight of the newborns.

Methods The current study is embedded in the PARA (Pregnancy-induced Amelioration of Rheumatoid Arthritis) study, a prospective nationwide study on RA and pregnancy. Patients with RA were seen before pregnancy, during every trimester and post partum. At all time points, maternal disease activity was calculated using Disease Activity Score 28 joints (DAS28-CRP). Serum levels of IL6 and TNF $\alpha$ levels were determined from the first trimester.

Results During the first trimester the mean serum level of TNF $\alpha$ and IL6 was $15.5 \mathrm{pg} / \mathrm{ml}$ (SD 6.8) and $7.5 \mathrm{pg} / \mathrm{ml}$ (SD 7.5), respectively. In $29 \%$ of sera, IL6 levels were below the detection limit $(2.0 \mathrm{pg} / \mathrm{ml})$. In linear regression on birth weight SDS (ie, birth weight adjusted for gestational age and sex), only disease activity was associated with lower birth weight $(p=035)$. No such 